ACCEPTED MANUSCRIPT

\title{
International comparison of noise in areal surface topography measurements
}

To cite this article before publication: Maxim Vanrusselt et al 2021 Surf. Topogr.: Metrol. Prop. in press https://doi.org/10.1088/2051-672X/abfa29

\section{Manuscript version: Accepted Manuscript}

Accepted Manuscript is "the version of the article accepted for publication including all changes made as a result of the peer review process, and which may also include the addition to the article by IOP Publishing of a header, an article ID, a cover sheet and/or an 'Accepted

Manuscript' watermark, but excluding any other editing, typesetting or other changes made by IOP Publishing and/or its licensors"

This Accepted Manuscript is @ 2021 IOP Publishing Ltd.

During the embargo period (the 12 month period from the publication of the Version of Record of this article), the Accepted Manuscript is fully protected by copyright and cannot be reused or reposted elsewhere.

As the Version of Record of this article is going to be / has been published on a subscription basis, this Accepted Manuscript is available for reuse under a CC BY-NC-ND 3.0 licence after the 12 month embargo period.

After the embargo period, everyone is permitted to use copy and redistribute this article for non-commercial purposes only, provided that they adhere to all the terms of the licence https://creativecommons.org/licences/by-nc-nd/3.0

Although reasonable endeavours have been taken to obtain all necessary permissions from third parties to include their copyrighted content within this article, their full citation and copyright line may not be present in this Accepted Manuscript version. Before using any content from this article, please refer to the Version of Record on IOPscience once published for full citation and copyright details, as permissions will likely be required. All third party content is fully copyright protected, unless specifically stated otherwise in the figure caption in the Version of Record.

View the article online for updates and enhancements. 


\title{
International comparison of noise in areal surface topography measurements
}

\author{
Maxim Vanrusselt ${ }^{1}$, Han Haitjema ${ }^{1}$, Richard Leach ${ }^{2}$ and Peter de Groot ${ }^{3}$ \\ ${ }^{1}$ Department of Mechanical Engineering, KU Leuven, Belgium \\ ${ }^{2}$ Manufacturing Metrology Team, Faculty of Engineering, University of Nottingham, UK \\ ${ }^{3}$ Zygo Corporation, Middlefield, United States \\ E-mail: han.haitjema@kuleuven.be
}

Received xxxxxx

Accepted for publication $\mathrm{xxxxxx}$

Published xxxxxx

\begin{abstract}
An international comparison of surface topography measurement noise was carried out. The comparison involved twelve optical surface topography instruments (focus variation instruments, confocal microscopes and coherence scanning interferometers) from six European research laboratories. The purpose of this comparison was to perform a practical test of the ISO definition and procedures for determining measurement noise on a variety of instruments, and to provide good practice guidance to the user community for quantifying, specifying and interpreting measurement noise. Despite taking steps to enable an optimal comparison by supplying a variety of specimens, where the 'best' could be selected and the measurement area was clearly defined, an appropriate comparison of the measurement noise beyond the actual measurement conditions was complex, as the measurement time, speed and lateral resolution were difficult to establish for most of the instruments. The results that could be obtained were: (i) the measurment noise $N_{M}$ can be reliably estimated from two measurements, (ii) noise may vary considerably between instruments, even when they have the same measurement principle, (iii) the noise in a practical measurement may be considerably higher than the noise with a levelled flat, and (iv) the "rms repeatability" has little correlation to the noise determined according to the ISO specification standard, and may lead to values that are more than a hundred times smaller.
\end{abstract}

Keywords: Surface analysis, Optical, Comparison, Measurement noise

\section{Introduction}

Areal surface topography measuring instruments are widely used for investigating the surfaces from conventional manufacturing methods, as well as from advanced precision manufacturing processes that are capable of producing surfaces with structural micro- and nanoscale topographic features $[1,2]$.

One of the key performance parameters for these instruments is the amount of random noise that is added to the measured topography. Instrument noise may be interpreted qualitatively as the smallest variations in surface heights that the instrument can detect above the noise level, given a specified number of data points and the acquisition time [3]. Historically, a wide variety of sometimes inconsistent terms and definitions related to noise have appeared in the literature and on manufacturer specification sheets $[4,5,6,7,8]$.

The recent ISO measurement performance verification and traceability framework for optical and tactile areal surface topography measuring instruments comprises material measures and calibration procedures $[9,10]$, and the ISO 25178-600 specification standard [11]. One of the defined metrological characteristics is the measurement noise, defined as the "...noise added to the output signal occurring during the normal use of the instrument." A closely related concept is the 
instrument noise, which is the lowest possible measurement noise value under ideal conditions [11]. These ideal conditions assume an experienced user, a laboratory environment and a test object selected to demonstrate best-achievable performance. Of equal interest is how the measurement noise changes with deviations from the ideal conditions, even for well controlled changes, such as the tip and tilt of the test part [12].

Procedures for calibrating measurement noise are currently being standardized in ISO/DIS 25178-700 [13]. These methods rely on repeated measurements of surface topography, using a type A uncertainty evaluation for random variations in the reported surface heights. These procedures have already been adopted by some manufacturers for routine verification of specification and for formal acceptance testing at installation sites [4]. The procedures have also been exercised by independent labs for objective determination of measurement noise as a function of time and part orientation, and as a way of validating instrument models [12]. However, the ISO procedures have not yet been widely adopted for different instrument types and measurement principles.

The present work is an international investigation of the state of the art in noise definitions, as defined in the latest ISO standards. An international comparison was organized, where parameters characterizing measurement and instrument noise were established on a variety of instruments in a range of laboratories. The purpose of this comparison was to road-test the ISO definition and procedures for measurement noise and to provide good practice guidance to the user community for quantifying, specifying and interpreting noise values.

The comparison involved twelve optical surface topography measuring instruments: four based on focus variation $(\mathrm{FV})$, four based on confocal microscopy $(\mathrm{CF})$ and four based on coherence scanning interferometry (CSI) (see [14] for the latest advances in these instruments). The measurements were performed in six laboratories in The Netherlands, Belgium, Denmark, Austria and UK. The comparison was organized by members of the Scientific Technical Committee 'Surfaces' of the International Academy for Production Engineering and by members of Working Group 16 'areal and profile surface texture' of ISO Technical Committee 21 and took place in 2019 to 2020.

\section{Description of the measurement artefacts}

The measurement artefacts consist of five optical flats and an example "typical" mechanical surface. The specifications are given below, where FLTt is the peak-to-valley flatness deviation, $R a$ is the arithmetical mean profile deviation and $S q$ is the areal root mean square height.

\section{Nominally flat surfaces}

- Mitutoyo optical flat, type 158-118, parallel glass, $45 \mathrm{~mm}$ diameter, $12 \mathrm{~mm}$ thickness, FLTt $<0.1 \mu \mathrm{m}$
- ZYGO flatness artefact, SiC, type 1776-666-012, $30 \mathrm{~mm}$ diameter, $S q<0.2 \mathrm{~nm}, F L T t<10 \mathrm{~nm}$

- $\quad$ Knight optical plane mirror, $25 \mathrm{~mm}$ diameter, $6 \mathrm{~mm}$ thickness, reflectance 99\%, FLTt $<0.06 \mu \mathrm{m}$

Nominally flat, but slightly roughened surfaces

- Alicona - IF right angle tool G1Vb1

- $\quad$ Mitutoyo - reference step artefact 02AKX920

Typical mechanical surface

- Rubert Microsurf 315: Surface Grinding, section: $R a \approx 0.4 \mu \mathrm{m}$

An auxiliary workpiece was supplied, consisting of a turned aluminum cylindrical workpiece with a $2^{\circ}$ wedge, that can be used to position the flat specimen at an angle of $2^{\circ}$ after alignment to a minimal tilt.

A variety of optical flats were made available in order to enable the selection of the optimum measurement conditions for each instrument. Depending on the instrument and the measurement principle, the noise may depend on the reflectivity, where $100 \%$ reflectivity is not by definition optimal, for example when the instrument is intentionally designed to function best over a range of expected reflectivities. For traditional FV measurements, a slight microscale surface texture must be present [8]. Figure 1 depicts the artefacts.

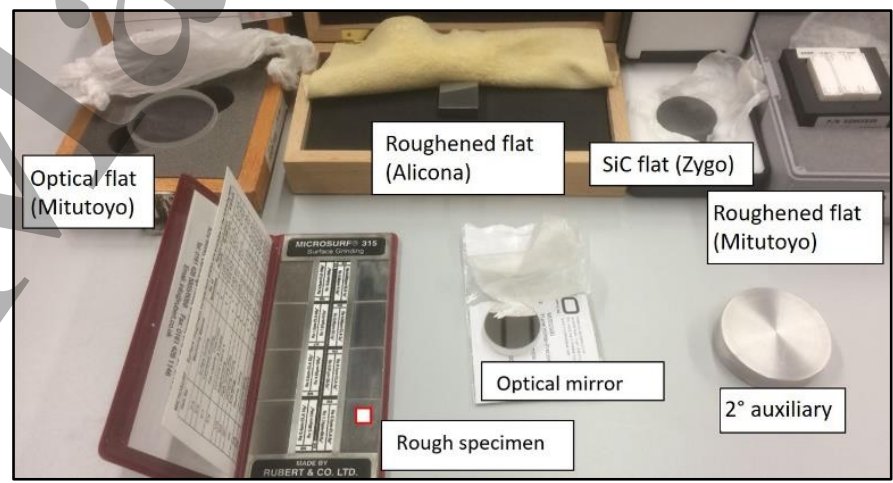

Figure 1. Artefacts used for the comparison

\section{Measurement procedure}

The participants were requested to select the optical flat that provides the optimum repeatability for the used instrument in optimally leveled position and to provide eight measurements of a $400 \mu \mathrm{m} \times 400 \mu \mathrm{m}$ area, or the smallest possible field of view that includes this area, for the following four cases:

1. Optimum nominally flat surface, optimally aligned with minimum tilt, eight repeated measurements without moving the artefact between the measurements. 
2. Optimum nominally flat surface, tilted $2^{\circ}$ around the $y$ axis, eight repeated measurements without moving the artefact between the measurements.

3. Rubert Microsurf 315 artefact, optimally aligned with minimum tilt, eight repeated measurements without moving the artefact between the measurements.

The motivation for selecting this set of measurement is that the first measurement gives the measurement noise that best approximates the instrument noise and it can be specified by manufacturers. The second measurement includes the effect of vibrations and linearity deviations in the height measurements. The third measurement gives an indication of the measurement noise where the surface contains local slopes and features as may appear in an actual measurement of a rough surface. As the noise is defined such that it is independent of the exact topography, defining an exact location for the measurements was considered not to be necessary. The participants were requested to supply the raw $z(x, y)$ topography coordinates, with as little filtering, interpolation or other data processing as the instrument allows, in digital form to the organizer.

\section{Measurement evaluation}

\subsection{Pre-processing of data}

Before further processing, for every measurement a $400 \mu \mathrm{m} \times 400 \mu \mathrm{m}$ region was selected from the data (if needed). The measurements contained no spikes and only two measurements contained missing data. For the calculation of the autocorrelation spectrum (see section 6.2) missing data were filled in by smooth interpolation to neighbouring valid data (this process is described in detail elsewhere [15]). The $z(x, y)$ data were levelled to the least-squares plane for every measurement.

\subsection{Evaluation of noise measurements}

The measurement noise $N_{M}$ defined in ISO 25178-600 is determined by the averaging method according to ISO/DIS 25178-700 using

$$
N_{M}=\sqrt{\frac{\sum_{i=1}^{n} S_{q}^{2}\left(z_{i}(x, y)-z_{m}(x, y)\right)}{n-1}},
$$

where $z_{m}$ is the mean value of the $z$-coordinates of the $n$ measurements, $n$ is the number of measurements $(n=8$ in this case) and $S_{q}$ is the root mean square height as defined in ISO 25178-2 [16]. This definition makes $N_{M}$ independent of the exact topography. In addition, the noise stability $S_{N M}$ is taken as the standard deviation of the noise estimates of the individual measurements:

$$
S_{N M}=\sqrt{\frac{\sum_{i=1}^{n}\left(N_{M}-N_{M i}\right)^{2}}{n-1}},
$$

where

$$
N_{M i}=\frac{S_{q}\left(z_{i}(x, y)-z_{m}(x, y)\right)}{\sqrt{1-n^{-1}}} .
$$

The noise stability can be regarded as a measure of the repeatability of the measurement noise. As an additional characteristic, the "repeatability of the rms" is sometimes used in instrument specifications, and is calculated as

$$
R_{r m s}=\sqrt{\frac{\sum_{i=1}^{n}\left(s_{q}\left(z_{i}(x, y)\right)-\frac{\sum_{i=1}^{n} s_{q}}{n}\right)^{2}}{n-1}} .
$$

\section{Results}

\subsection{Measurement noise results}

The measurement noise results, calculated using equation (1), for the participants data are given in Figure 2. The noise is calculated for the aligned flat, the tilted flat and for the Rubert artefact; all with eight repeated measurements on the same position. The used instruments are grouped by type, though it must be emphasized that this grouping does not imply that these measurement principles have fundamental noise limitations as indicated in the figure. Measuring instruments are systems that consist of mechanical and optical elements and software that may be as distinctive as the measurement principle. The need to use a logarithmic vertical scale to usefully present the results is illustrative of the degree of variation between the instruments and measurements, even for instruments using the same measurement principle.

\subsection{Repeatability of the measurement noise}

In ISO/DIS 25178-700, the default procedure for obtaining the measurement noise parameter $N_{M}$ is to take two measurements, which simplifies equation (1) to

$$
\begin{gathered}
N_{M}=\sqrt{\sum_{i=1}^{2} S_{q}^{2}\left(z_{i}(x, y)-\frac{z_{1}(x, y)+z_{2}(x, y)}{2}\right)} \\
=\sqrt{2 S_{q}^{2}\left(\frac{z_{1}(x, y)-z_{2}(x, y)}{2}\right)}=S_{q}\left(z_{2}(x, y)-z_{1}(x, y)\right) / \sqrt{2}
\end{gathered}
$$

Equation (5) implies that $N_{M}$ can be obtained from the difference of two measurements, something that is commonly possible in instrument software. We investigated whether application of (5) gives a viable evaluation of $N_{M}$ considering the stability of the obtained $N_{M}$-values. 


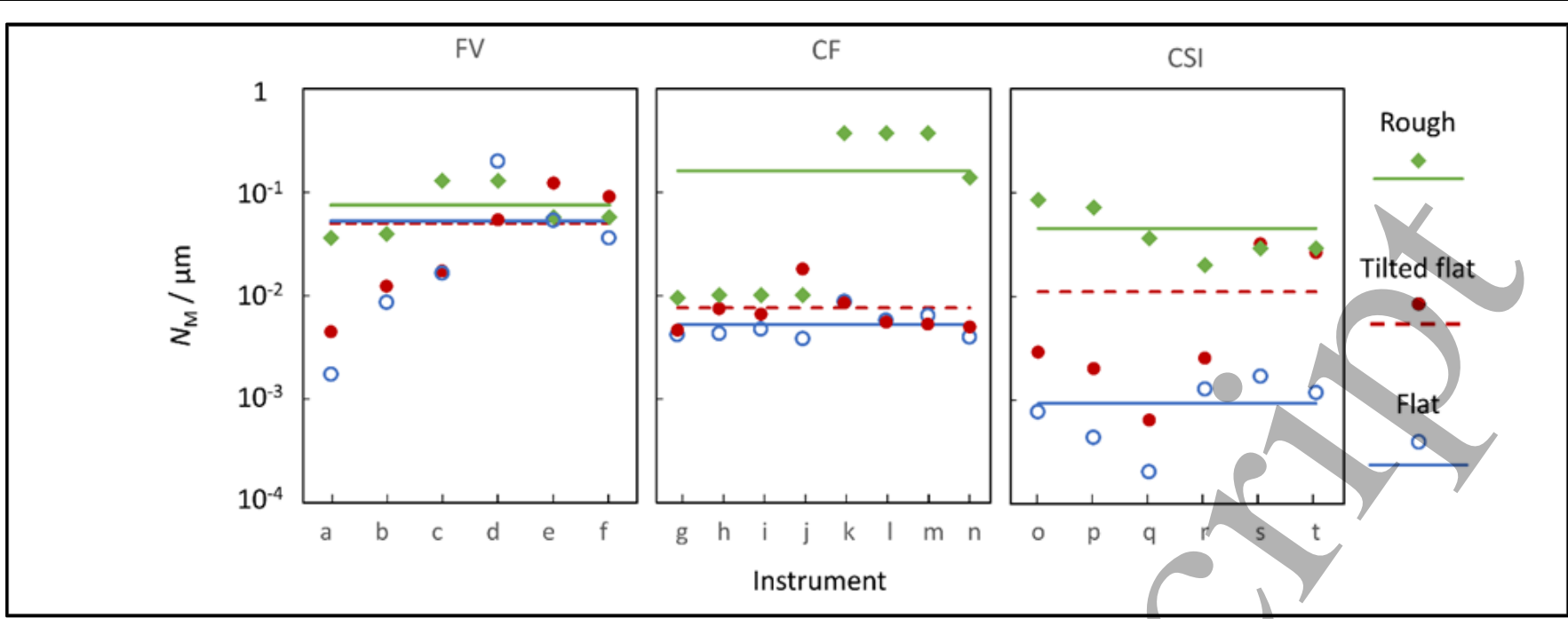

Figure 2. Measurement noise $N_{M}$ for different instruments (a-t) when measuring a leveled flat artefact ('Flat'-blue), a tilted flat artefact ('Tilted flat'-red) artefact and the moderately rough Rubert artefact ('Rough'-green) measured by optical topography measuring instruments, based on focus variation ( $\mathrm{FV}, \mathrm{a}-\mathrm{f})$, confocal microscopy $(\mathrm{CF}, \mathrm{g}-\mathrm{n})$ and coherence scanning interferometry (CSI,o-t) respectively. Measurements $\mathrm{c}$ and $\mathrm{d}$ are taken with the same instrument, but with a different slightly roughened flat; for measurements e and $\mathrm{f}$ the same holds. Measurements $(\mathrm{h}, \mathrm{i}, \mathrm{j}),(\mathrm{k}, \mathrm{l}, \mathrm{m})$ and $(\mathrm{s}, \mathrm{t})$ are taken with the same instrument, but using a different optical flat (h,k,s: glass; j,m,t: SiC; i,l: mirror). Therefore, these same instruments show the same value for the 'Rough' measurement. Measurements $\mathrm{o}$ and $\mathrm{p}$ are taken using a different measuring mode of the same instrument. The horizontal lines indicate the mean within the group of instruments.

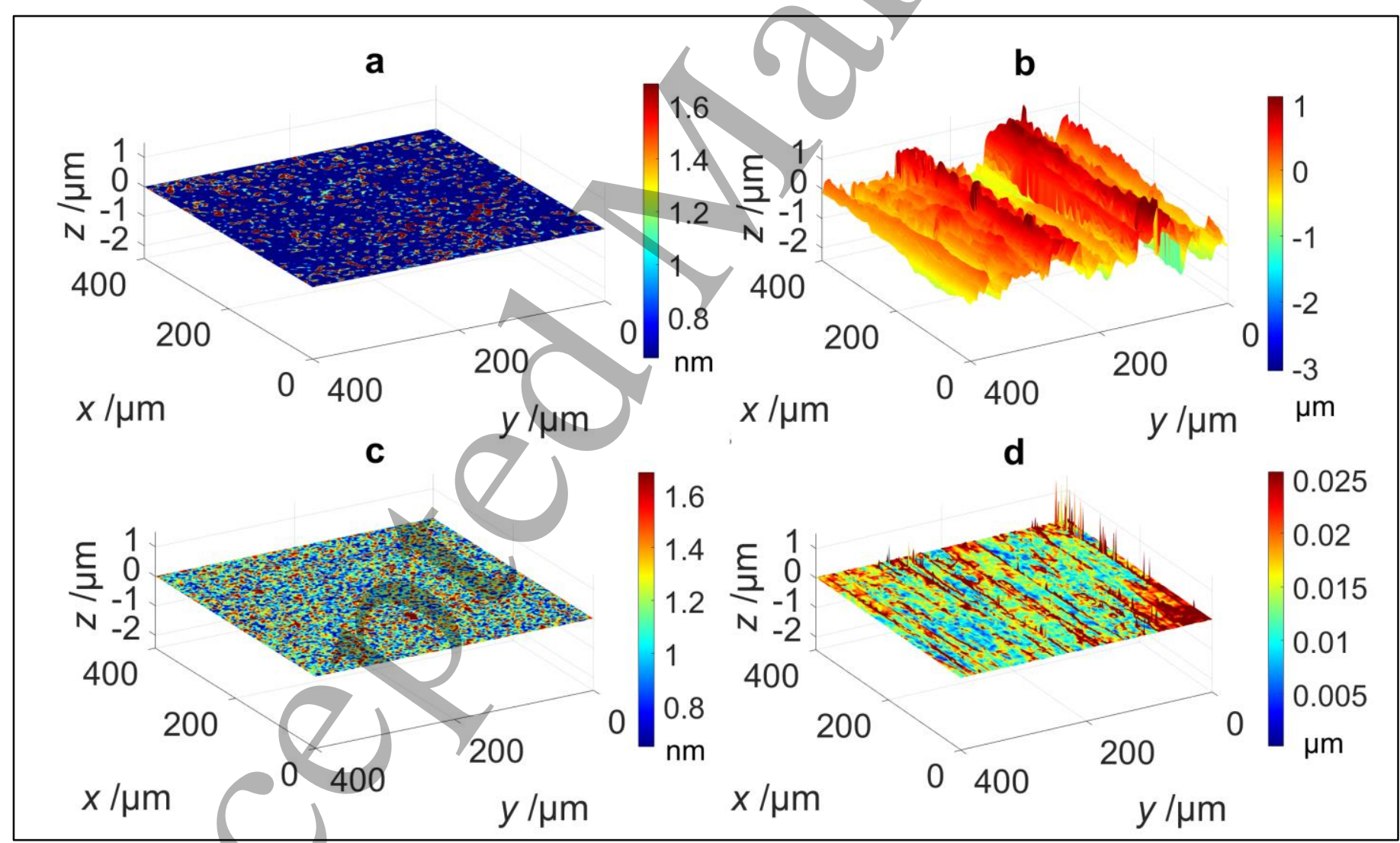

Figure 3. Measurement noise map for a coherence scanning interferometry measuring instrument. a. topography of a levelled flat measurement, b. topography of a moderately rough Rubert artefact, c. noise map of a levelled flat measurement, d. noise map of a moderately rough Rubert artefact.

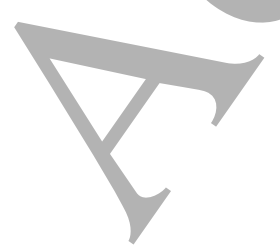


From the eight measurements taken for every condition in this comparison, the noise stability $s_{N M}$ was obtained; this value gives a measure for the repeatability of the measurement noise when equation (5) is used. The relative noise stability $s_{N M} / N_{M}$ varied between the measurements but rarely exceeded $30 \%$ of the measured $N_{M}$ value. The mean relative noise stability was $28 \%$ for the focus variation measurements, $16 \%$ for the confocal measurements and $11 \%$ for the CSI measurements. This implies that using equation (5) gives an expected repeatability that is characterised by a relative standard deviation of $20 \%, 11 \%$ and $8 \%$ for FV, CF and CSI measurements respectively. From these values, it can be concluded that the default ISO procedure gives a viable estimation of the measurement noise when a standard uncertainty of this value within $20 \%$ is considered appropriate.

\subsection{Relationship between measurement noise and instrument noise}

In ISO 25168-600, the instrument noise $N_{I}$ is defined as the internal noise added to the output signal caused by the instrument if ideally placed in a noise-free environment, whereas the measurement noise $N_{M}$ is defined as the noise added to the output signal occurring during the normal use of the instrument. For measurement instrument specifications, it is recommended in ISO/DIS 25178-700 to use an optimally aligned optical flat. The reason behind this choice is that this measurement is supposed to be least sensitive for the noise contributors that distinguish the specimen- and environmentdependent measurement noise from the instrument noise. This means that this is the measurement that most closely approaches the instrument noise in practice. In Figure 2, it is shown that the measurement noise determined by measuring an optical flat when aligned for minimal tilt is consistently lower than when intentionally tilting that flat, and can be several orders of magnitude lower than when measuring a non-flat artefact. Similar effects have been reported for CSI measurements [12].

The effect of the actually used flat is limited for CSI and CF measurements, as shown by comparing measurements $(\mathrm{h}, \mathrm{i}, \mathrm{j}),(\mathrm{k}, \mathrm{l}, \mathrm{m})$ and $(\mathrm{s}, \mathrm{t})$. On the other hand, the significant differences between measurements $c$ and $d$ as well as between $\mathrm{e}$ and $\mathrm{f}$ shows quite some dependence of the noise on the actual 'roughened flat' surface used here for FV measurement and raises a question regarding whether the used surface and measurement conditions (e.g. the lighting, resolution setting) were optimal for this case (given the measurement area and used objective).

The increase in noise when tilting the surface illustrates the dependence of a repeatable and smooth scanning and the increased influence of vibration. Especially measurements $s$ and $t$ appear to suffer from vibration.
In general, the measurement of the rough surface results in more noise in areas where the local gradients and corresponding low intensity values also have an effect.

\subsection{Location-dependent noise}

In the definition of $N_{M}$, it is more or less implied that its value is constant over the surface. For flat or smooth surfaces, this was commonly the case, however, for the Rubert artefact, significant variation over the surface was observed for several instruments. Figure 3 shows the location-dependent noise, i.e. the standard deviation in every $z(x, y)$-coordinate, over the surface for both the levelled flat and the Rubert artefact for a CSI instrument. Figure 3 shows a homogeneous characteristic for the noise from the measured flat, but for the rough artefact, the noise may be significantly higher in regions having irregularities or gradients within a small region on the surface. It is especially these regions that cause the high $N_{M}$ values for the Rubert artefact compared to the optical flat measurements in Figure 2. Position-dependent noise has been studied in more detail elsewhere [17].

\subsection{Repeatability of the rms}

A term that some manufacturers use to specify surface topography instrument noise is the repeatability of the rms or similar, as defined in equation (4). The shortcomings of this definition can be argued from first principles: the parameter depends fundamentally on the noise variation rather than the noise itself, and, where the noise combines with the $S q$-value of the surface itself, it gives a lower value for a higher level of surface texture and/or flatness deviation. As an illustration, the $R_{r m s}$ is plotted against $N_{M}$ for all measurements taken in this comparison. The results are displayed in Figure 4. This figure shows that the $R_{r m s}$ ranges from the same order of magnitude as $N_{M}$ to more than two orders of magnitude smaller than $N_{M}$, and logarithmic scales must be used to depict the wide scatter of results. In Figure 4, the measurement instrument types are distinguished, illustrating that the lack of a consistent relationship holds for all instruments tested here. Also, distinguishing between the object (flat, tilted flat, rough) showed no systematic effects. From this result, it can be confirmed from practical measurements that the repeatability of the rms shows little correlation with the measurement noise and is an inappropriate way for specifying measurement noise, as has been noted elsewhere [4].

\section{Measurement speed and measured points}

Areal surface topography measuring instruments are no different from any other kind of sensor, in that a specification of random noise has little meaning without a statement regarding the data acquisition bandwidth [7].

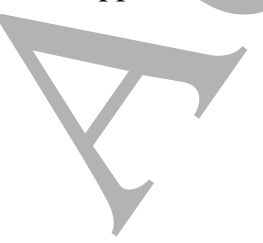




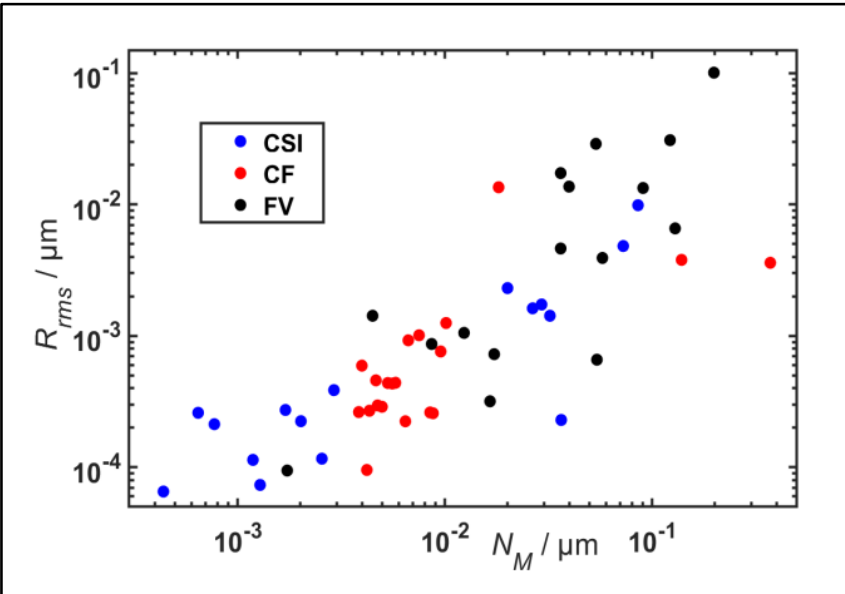

Figure 4. Relationship between measurement noise and repeatability of the rms obtained by all measurements in this comparison.

In the draft ISO/DIS 25178-700 standard, it is advised that "...an instrument noise specification shall include the relevant data acquisition time, the number of independent data points, and any default filtering of the surface topography." While this additional information is of interest regardless of the source of measurement variability, if the dominant noise sources can be considered as random, the noise $N_{M}$ can be improved at the cost of longer measurement times $t$ or a reduction in the number $P$ of independent, uncorrelated image points [3,7].

These two parameters, data acquisition time and the number of independent data points in the field of view, affect the measurement noise level and should be taken into account when comparing $N_{M}$ values. One approach to quantifying these effects is by a single noise density parameter $\eta_{M}$ defined as:

$$
\eta_{M}=N_{M} \sqrt{\frac{t}{P}}
$$

Noise density is a common way to specify a wide variety of sensors and detectors for which the data acquisition bandwidth is of importance [7,18]. Therefore harmonizing the $N_{M}$ values into $\eta_{M}$ values should enable noise comparisons between different instruments, where different measurement times, filtering and lateral resolution are accounted for.

\subsection{Measurement speed and data acquisition rate}

As the time $t$ required for data acquisition is a function of the total surface height measurement range, the data acquisition rate can be expressed as the measurement range for a given $t$ [3]. However, for most instruments in this study, it was not possible to obtain a useful value for $t$ that could be used as an estimation for $\eta_{M}$ in equation (6). Most instruments measured the levelled flat in approximately $6 \mathrm{~s}$ for a $50 \mu \mathrm{m}$ range, with two outliers: instrument $\mathrm{n}$ taking $66 \mathrm{~s}$, and instrument q measuring the flat artefact in $0.07 \mathrm{~s}$. This would make $\eta_{M}$ for measurement $\mathrm{n}$ a factor of $3.5 \times$ larger and for measurement $\mathrm{q}$ a factor of $10 \times$ lower compared to the other measurements. A conclusion here is that it would be helpful for instrument manufacturers to include either in user manuals or directly in the software, the necessary information to determine the data acquisition speed, and how this may be influenced by data averaging, oversampling or other means to improve the noise level at the cost of a longer measurement time.

\subsection{Number of independent measurements}

The number of independent measured points can be estimated as the measured area divided by the square of the topographic spatial resolution $W_{R}$. As the determination of $W_{R}$ was not a part of this comparison and these data were not available, two methods were used to estimate the number of independent measured points $P$ : using the noise variation and using autocorrelation.

Assuming a Gaussian distributed noise, an equation for the standard deviation in the standard deviation can be written as [19]:

$$
P_{n v}=0.5\left(\frac{N_{M}}{s_{N M}}\right)^{2}+1
$$

Useful application of this equation requires homogeneity of the noise over the surface and the absence of any correlation due to, e.g., vibration, which cannot be guaranteed, even with an optimally aligned flat surface.

If it can be assumed that the measured topography noise is spatially independent of the topography coordinates, $P$ can be estimated from the autocorrelation length using

$$
P_{a c}=\frac{l_{x} l_{y}}{P_{a l x} P_{a l y}}
$$

where $l_{x}, l_{y}$ are the measurement lengths in the $x$ and $y$ directions, and $P_{a l x}, P_{a l y}$ are the correlation lengths in the $x$ and $y$ directions respectively. Equation (8) captures implicit or explicit software filtering of high spatial frequencies and the pixel size, but not the limitations from the optical system, such as the numerical aperture of the objective. Figure 5 shows the autocorrelation spectrum for a FV measurement $(\mathrm{g})$ and a CSI measurement $(\mathrm{t})$, taken as the mean autocorrelation spectrum of the 'noise topographies' $z_{i}(x, y)-z_{m}(x, y)$ as is also used in equation (3). Clearly, the FV measurement in Figure 5 shows a much larger autocorrelation length than the CSI measurement, giving $P_{a c}=28$ and $P_{a c}=6400$ respectively. However, applying equation (8) for these measurements gives $P_{n v}=18$ and $P_{n v}=2300$ respectively. 


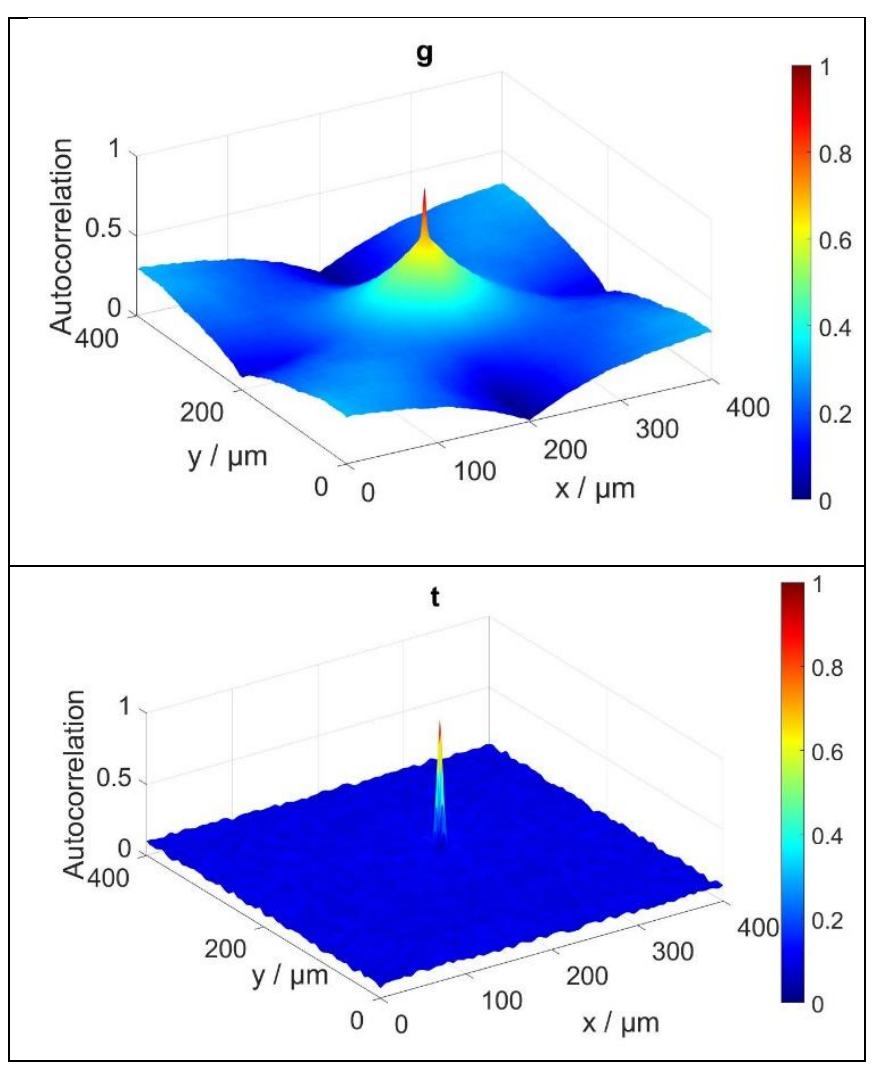

Figure 5. Autocorrelation functions for the noise measurements $\mathrm{g}$ and $\mathrm{t}$

Although both estimations of $P$ show a similar trend, the agreement between $P_{n v}$ and $P_{a c}$ was in this case insufficient to come to a useful evaluation and comparison of $\eta_{M}$-values. This was also the case for most other measurements, which makes it difficult to normalize the $N_{M}$ values to $\eta_{M}$ values without explicit measurement of the topographic spatial resolution $W_{R}$.

\section{Concluding remarks}

A comparison of measurement noise was made for a wide range of surface topography measurements. The measurement noise parameter $N_{M}$ could be determined in all cases and it was confirmed that the default method for obtaining the measurement noise by taking just the difference of two topographies is a viable method.

In the design of the comparison, we supplied a variety of specimens where the 'best' could be selected, and defined the measurement area. However, an appropriate comparison of the noise was complicated by factors such as the measurement time and the actual lateral resolution. For the data acquisition time, this was not always clear, nor was it clear how this time could be adjusted to improve the noise level. Attempts to determine the number of independent data points by correlation length and noise variation showed some promise, but were not satisfactory enough for us to claim that we have established an appropriate method for doing this.

Given these factors, it is perhaps not surprising that the measurements results varied over orders of magnitude, depending on the measurement speed, and software filtering, in addition to differences in measurement principle and instrument design. A clear conclusion is that noise specifications should include information about the measured area, the effective lateral resolution, the measuring speed, default filtering and other quantities that influence the measurement noise. While these considerations have been at least partly adopted by some instrument manufacturers and testing laboratories, there remains more work to do to advance the adoption of the ISO definitions of measurement and instrument noise, as well as the recommended procedures.

The comparison also raised the question of defining or obtaining an optimum flat-like surface that gives minimum noise for a FV instrument. It is confirmed and illustrated that the measurement noise in a practical measurement may be considerably higher than the noise for a levelled flat, and it may vary significantly over the surface.

\section{Acknowledgements}

We would like to thank all participants of the comparison for the time and effort that they invested in the measurements: Lucas Redlarski and Adriaan Zuiderweg (Mitutoyo RCE, The Netherlands), Rong Su, Adam Thompson and Mingyu Liu (University of Nottingham, UK), Jona Gladines and Johan Blom (University of Antwerp, Belgium), Gerald Haueisen and Franz Helmli (AliconaBruker, Austria), and Yang Zhang (Technical University of Denmark).

\section{References}

[1] Zhang S J, To S, Wang S J, Zhu Z W 2015 A review of surface roughness generation in ultra-precision machining Int. J. Mach. Tools Manufac. 91 76-95

[2] Brinksmeier E, Karpuschewski B, Yan J, Schönemann L 2020 Manufacturing of multiscale structured surfaces Ann. CIRP 69 717-739

[3] de Groot P, DiSciacca J 2018 Surface-height measurement noise in interference microscopy Proc. SPIE 10749107490

[4] de Groot P 2014 Progress in the specification of optical instruments for the measurement of surface form and texture Proc. SPIE 9110 91100M

[5] Haitjema H, Morel MAA 2005 Noise bias removal in profile measurements Measurement 38 21-29

[6] Giusca C L, Leach R K, Helary F, Gutauskas T, Nimishakavi L 2012 Calibration of the scales of areal surface topographymeasuring instruments: Part 1. Measurement noise and residual flatness Meas. Sci. Technol. 23035008 
[7] de Groot P, DiSciacca J 2020 Definition and evaluation of topography measurement noise in optical instruments Opt. Eng. 59064110

[8] Helmli F Focus variation instruments. In: Leach R K Optical Measurement of Surface Topography (Springer)

[9] Leach R K, Haitjema H, Su R, Thompson A 2021 Metrological characteristics for the calibration of surface topography measuring instruments: a review Meas. Sci. Technol. 32032001

[10] Leach R K, Giusca C L, Haitjema H, Evans C, Jiang X 2015 Calibration and verification of areal surface texture measuring instruments Ann CIRP 64 797-813

[11] ISO 25178-600 2019 Geometric Product Specifications (GPS) -Surface texture: Areal-Part 600:Metrological characteristics for areal topography measuring instruments, International Organization for Standardization, Geneva, Switzerland.

[12] Gomez C, Su R, de Groot P, Leach RK 2020 Noise reduction in coherence scanning interferometry for surface topography measurement Nanomanufacturing and Metrology 3, 68-76

[13] ISO-DIS 25178-700 2020 Geometric Product Specifications (GPS) -Surface texture: Areal-Part 700: Calibration, adjustment and verification of areal topography measuring instruments, International Organization for Standardization, Geneva, Switzerland.

[14] Leach R K 2020 Advances in Optical Surface Texture Metrology (IOPP)

[15] John D'Errico 2020 inpaint_nans (https://www.mathworks.com/matlabcentral/fileexchange/4551inpaint_nans), MATLAB Central File Exchange. Retrieved March 13, 2021.

[16] ISO 25178-2 2012 Geometric Product Specifications (GPS) Surface texture: Areal-Part 2: Terms, definitions and surface texture parameters, International Organization for Standardization, Geneva, Switzerland

[17] Venditti K 2020 Design for metrology of free form optics, MSc thesis, ProQuest LLC, Ann Arbor, USA

[18] Usher M J 1974 Noise and bandwidth J.Phys. E Sci. Instrum. 7 957-961

[19] Squires G L 2001 Practical Physics (Cambridge University Press)

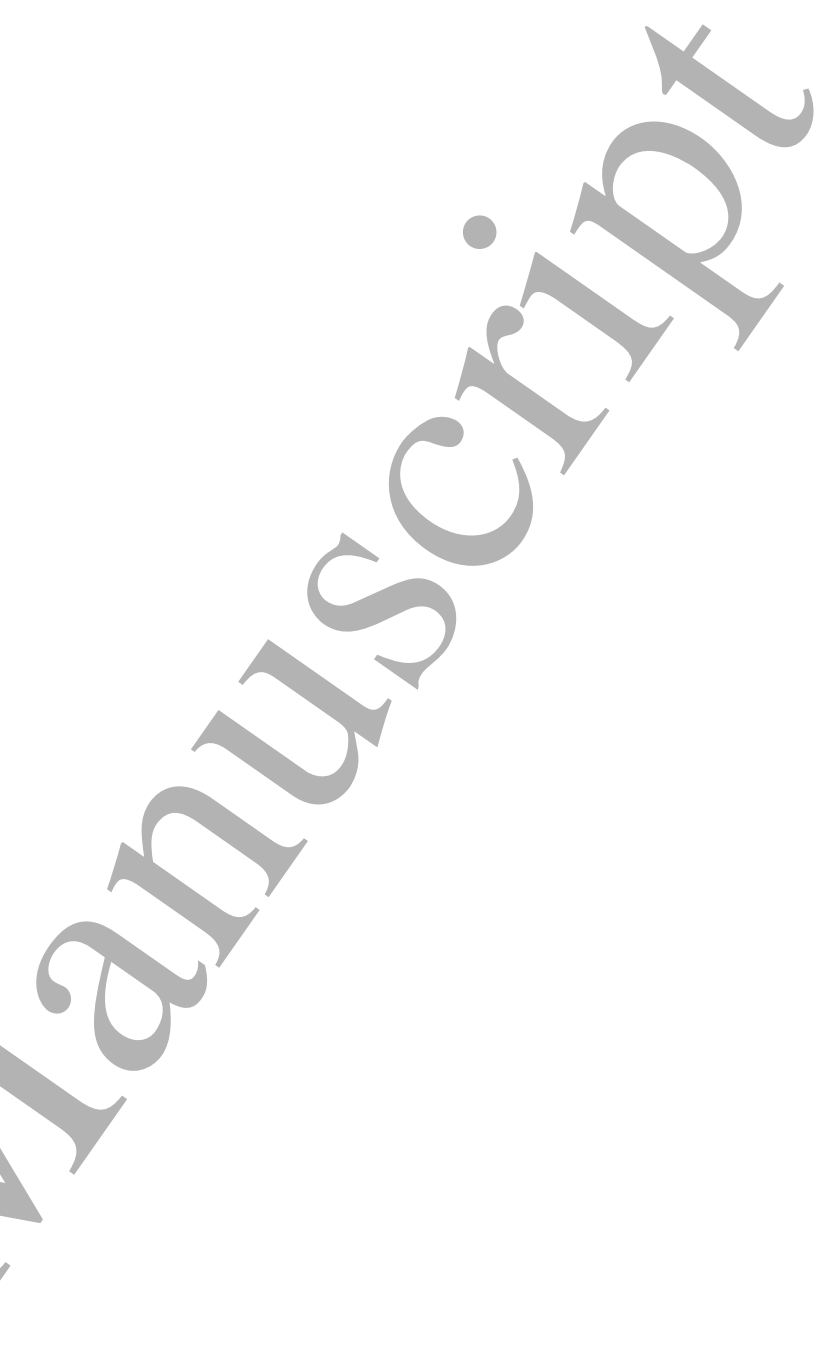

